\title{
Crystal structure of (18-crown-6)bis(tetrahydrofuran)potassium bis(2,3,4,5-tetramethylphospholyl)bis(borohydride)neodymium(III), $\left[\mathrm{K}\left(\mathrm{C}_{12} \mathrm{H}_{24} \mathrm{O}_{6}\right)\left(\mathrm{C}_{4} \mathrm{H}_{8} \mathrm{O}\right)_{2}\right]\left\{\left[\mathrm{C}_{4}\left(\mathrm{CH}_{3}\right)_{4} \mathrm{P}\right]_{2} \mathrm{Nd}\left(\mathrm{BH}_{4}\right)_{2}\right\}$
}

\author{
S. Cendrowski-Guillaume, G. Le Gland, M. Ephritikhine and M. Nierlich* \\ CEA Saclay. DSM/DRECAM/SCM. Bât. 125. F-91191 Gif-sur-Yvette Cedex. France
}

Received September 9. 2001, accepted December 18, 2001; CCDC-No. $1267 / 722$

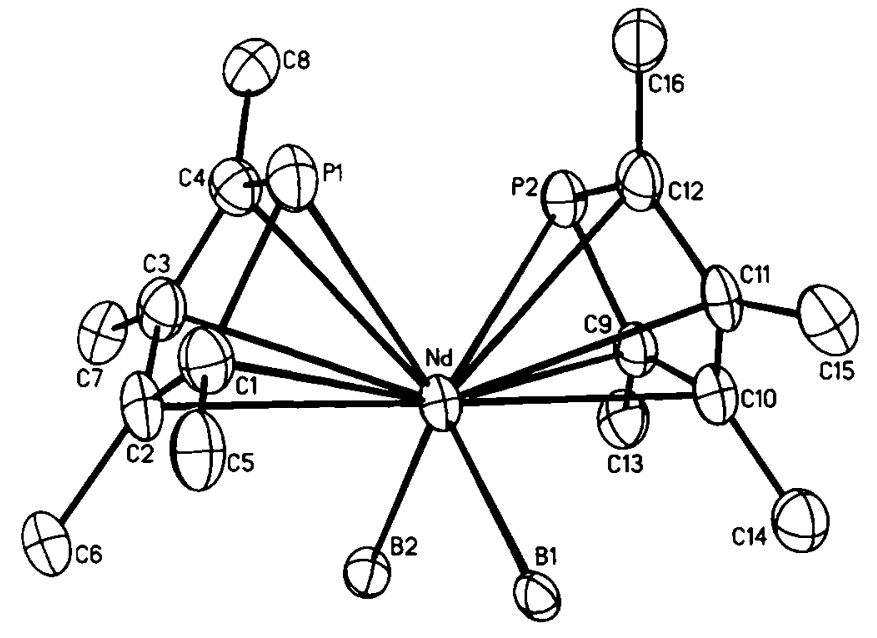

Abstract

$\mathrm{C}_{36} \mathrm{H}_{72} \mathrm{~B}_{2} \mathrm{KNdO}_{8} \mathrm{P}_{2}$, triclinic, $P \overline{\mathrm{I}}$ (No. 2), $a=10.489(2) \AA$, $b=11.784(2) \AA, c=19.103(4) \AA, \alpha=98.29(3)^{\circ}, \beta=94.07(3)^{\circ}$, $\gamma=104.26(3)^{\circ}, V=2250.5 \AA^{3}, Z=2, R_{\mathrm{gt}}(F)=0.069$, $w R_{\text {ref }}\left(F^{2}\right)=0.194, T=123 \mathrm{~K}$.

\section{Source of material}

Addition of 18 -crown-6 (1.7 equivalents, $143 \mu \mathrm{mol})$ to a THF $(20 \mathrm{~mL})$ solution of $[\mathrm{K}(\mathrm{THF})]\left[\mathrm{Nd}\left(\mathrm{PC}_{4} \mathrm{Me}_{4}\right)_{2}\left(\mathrm{BH}_{4}\right)_{2}\right](47.3 \mathrm{mg}$, $84 \mu \mathrm{mol})$ quantitatively gave the title compound. The product was recrystallized from a THF-pentane mixture to afford light blue crystals within 7 days. The sample was also characterized by NMR and IR spectroscopy. Spectroscopic data are included in the deposited CIF-file.

\section{Experimental details}

Hydrogen atoms (except $\mathrm{H}$ atoms of $\mathrm{BH}_{4}$ ) were introduced at calculated positions as riding atoms, with an isotropic displacement parameter equal to I.2 $\left(\mathrm{CH}, \mathrm{CH}_{2}\right)$ or $1.5\left(\mathrm{CH}_{3}\right)$ times that of the parent atom. The hydrogen atoms of $\mathrm{BH}_{4}$ were not found.

\section{Discussion}

Organometallic borohydrides complexes of neodymium are quite rare $[1,5]$. Bisborohydride derivatives include the cyclopentadienyl substituted species $\left(\mathrm{C}_{5} \mathrm{H}^{i} \mathrm{Pr}_{4}\right) \mathrm{Nd}\left(\mathrm{BH}_{4}\right)_{2}$ [1] and $\left[\mathrm{K}\left(18 \text {-crown-6) }\left\{\left(\mathrm{C}_{13} \mathrm{H}_{8}\right) \mathrm{CPh}_{2}\left(\mathrm{C}_{5} \mathrm{H}_{4}\right) \mathrm{Nd}\left(\mathrm{BH}_{4}\right)_{2}\right\}\right]_{2}\right.$ [3] while the monoborohydride compounds, in addition to $\left(\mathrm{C}_{5} \mathrm{H}_{4} \mathrm{CH}_{2} \mathrm{CH}_{2} \mathrm{OMe}\right)_{2} \mathrm{Nd}\left(\mathrm{BH}_{4}\right)$ [4], are essentially limited to the cyclooctatetraenyl derivatives $\left(\eta-\mathrm{C}_{8} \mathrm{H}_{8}\right) \mathrm{Nd}\left(\mathrm{BH}_{4}\right)(\mathrm{THF})_{2}$ and
$\left(\eta-\mathrm{C}_{5} \mathrm{Me}_{5}\right) \mathrm{Nd}\left(\mathrm{BH}_{4}\right)_{2}(\mathrm{THF})_{2}[2,5]$. Such borohydrides of neodymium(III) revealed themselves as versatile precursors for the synthesis of previously inaccessible compounds such as the cationic derivatives $\left[\left(\eta-\mathrm{C}_{8} \mathrm{H}_{8}\right) \mathrm{Nd}(\mathrm{L})_{\mathbf{x}}\right]\left[\mathrm{BPh}_{4}\right]\left(\mathrm{L}=\mathrm{THF}_{4}\right.$, $\mathrm{HMPA}_{3}$ ) $[5,6]$. Although a few bis(phospholyl)lanthanide(III) examples are known [7,8], the complex we isolated, [ $\mathrm{K}(\mathrm{THF})]\left[\left(\mathrm{PC}_{4} \mathrm{Me}_{4}\right)_{2} \mathrm{Nd}\left(\mathrm{BH}_{4}\right)_{2}\right]$ [2], is the first mixed borohydride-phospholyl derivative of a $4 f$ element. Addition of a crown ether to that product allowed the synthesis of the parent complex [K(18-crown-6)(THF) $]\left[\left(\mathrm{PC}_{4} \mathrm{Me}_{4}\right)_{2} \mathrm{Nd}\left(\mathrm{BH}_{4}\right)_{2}\right]$, the crystal structure of which has been determined and that is reported in this paper.

The structure of $\left[\mathrm{K}(18-\mathrm{crown}-6)(\mathrm{THF})_{2}\right]\left[\left(\mathrm{PC}_{4} \mathrm{Me}_{4}\right)_{2} \mathrm{Nd}\left(\mathrm{BH}_{4}\right)_{2}\right]$ reveals molecular cation and anion pairs. The figure shows the molecular anion. The potassium ion, which is encapsulated into the crown ether and surrounded by two coordinated THF, displays the expected coordination. The molecular anion adopts a distorted tetrahedral configuration with the following relevant angles $\angle \mathrm{B} 1-\mathrm{Nd}-\mathrm{B} 2=98.7(3)^{\circ}, \angle \mathrm{P} 1^{*}-\mathrm{Nd}-\mathrm{P} 2^{*}=128.7(7)^{\circ}$ and the mean value for $\angle \mathrm{B}-\mathrm{Nd}-\mathrm{P}^{*}=106.3(7)^{\circ}$, ranging from $105.6(2)$ to $107.3(2)^{\circ}\left(P^{*}=\right.$ phospholyl centroid $)$. The phospholyl ligands are bound to the metal in an $\eta^{5}$ mode. Both phospholyl rings are planar (within $0.031 \AA$ and $0.021 \AA$ for phospholyls "P1" and "P2" respectively) with average $\mathrm{Nd}-\mathrm{C}$ bond distance of $2.87(2) \AA, N d-P 1$ and Nd-P2 bond distances of $2.982(3) \AA$ and 2.995(3) $\AA$ and Nd-phospholyl centroid of 2.579(3) $\AA$ and 2.595(3) $\AA$ for "P1 " and "P2 ", respectively. These distances are comparable to those observed in the only other crystallographically characterized bisphospholyl lanthanide(III) complex, $\left.\left(\left[\eta^{5}-\mathrm{C}_{4} \mathrm{Me}_{4} \mathrm{P}\right) \mathrm{Sm}\left(\mu_{3}-\mathrm{Cl}\right)_{2}\left(\mu: \eta^{5}, \eta^{1}-\mathrm{C}_{4} \mathrm{Me}_{4} \mathrm{P}\right) \mathrm{K}\left(\mathrm{Et}_{2} \mathrm{O}\right)\right]_{\infty}\right),(d(\mathrm{Sm}-\mathrm{C})$ $=2.78(2) \AA, d(\mathrm{Sm}-\mathrm{P})=2.916(11) \AA)[9]$. The Nd-borohydride ligands distances ranging from $2.588(7) \AA$ to $2.670(5) \AA$ and averaging to $2.63(5) \AA$ agree well with the short distances observed for a tridentate binding mode of the $\mathrm{BH}_{4}$ ligand in $\left(\mathrm{C}_{5} \mathrm{H}^{i} \mathrm{Pr}_{4}\right) \mathrm{Nd}\left(\mathrm{BH}_{4}\right)_{2}$ (mean $d(\mathrm{Nd}-\mathrm{B})=2.600(8) \AA$ ) [1], $\left(\mathrm{C}_{5} \mathrm{H}_{4} \mathrm{CH}_{2} \mathrm{CH}_{2} \mathrm{OMe}\right)_{2} \mathrm{Nd}\left(\mathrm{BH}_{4}\right)(2.664(25) \AA)[4]$ or in $\left[\mathrm{K}\left(18 \text {-crown-6) }\left\{\left(\mathrm{C}_{13} \mathrm{H}_{8}\right) \mathrm{CPh}_{2}\left(\mathrm{C}_{5} \mathrm{H}_{4}\right) \mathrm{Nd}\left(\mathrm{BH}_{4}\right)_{2}\right\}\right]_{2} \cdot \mathrm{C}_{4} \mathrm{H}_{8} \mathrm{O}_{2}\right.$ (mean $d(\mathrm{Nd}-\mathrm{B})=2.632(9) \AA)$ [3]. This value is however much shorter than that measured in the dimeric complex $\left[\left(\eta-\mathrm{C}_{8} \mathrm{H}_{8}\right) \mathrm{Nd}\left(\mu_{3}-\mathrm{H}\right)_{2} \mathrm{~B}\left(\mu_{2}-\mathrm{H}\right)_{2} \text { (THF) }\right]_{2}(2.875(6) \AA$ and $2.941(6) \AA)$ [5]. The infrared data of our complex confirm the tridentate binding mode observed crystallographically $[10,11]$.

* Correspondence author (e-mail: nierlich@drecam.cea.fr) 
Table 1. Data collection and handling.

\begin{tabular}{ll}
\hline Crystal: & sky-blue, irregular, \\
& size $0.06 \times 0.10 \times 0.10 \mathrm{~mm}$ \\
Wavelength: & Mo $K_{\alpha}$ radiation $(0.71073 \AA)$ \\
$\mu:$ & $13.60 \mathrm{~cm}^{-1}$ \\
Diffractometer, scan mode: & Nonius Kappa-CCD, $\Delta \phi=2^{\circ}$ \\
$2 \theta_{\text {max }}$ & $49.34^{\circ}$ \\
$N(h k l)_{\text {measured, }} N(h k l)_{\text {unique: }}:$ & 13567,7004 \\
Criterion for $I_{\text {obs, }} N(h k l)_{\text {gi: }}:$ & $I_{\text {obs }}>2 \sigma\left(I_{\text {obs }}\right), 4318$ \\
$N(\text { param })_{\text {refined: }}$ & 454 \\
Program: & SHELXTL $[12]$
\end{tabular}

Table 2. Atomic coordinates and displacement parameters (in $\AA^{2}$ ).

\begin{tabular}{llllll}
\hline Atom & Site & $\boldsymbol{x}$ & $y$ & $z$ & $U_{\text {iso }}$ \\
\hline H(5A) & $2 i$ & 0.6400 & 0.9388 & 0.5002 & 0.076 \\
H(5B) & $2 i$ & 0.5445 & 0.8428 & 0.5344 & 0.076 \\
H(5C) & $2 i$ & 0.6976 & 0.8575 & 0.5448 & 0.076 \\
H(6A) & $2 i$ & 0.9249 & 1.0950 & 0.5960 & 0.080 \\
H(6B) & $2 i$ & 0.8889 & 0.9614 & 0.6056 & 0.080 \\
H(6C) & $2 i$ & 0.9572 & 1.0608 & 0.6703 & 0.080 \\
H(7A) & $2 i$ & 0.8895 & 1.2887 & 0.7363 & 0.082 \\
H(7B) & $2 i$ & 0.9283 & 1.1795 & 0.7613 & 0.082 \\
H(7C) & $2 i$ & 0.8294 & 1.2380 & 0.8017 & 0.082 \\
H(8A) & $2 i$ & 0.5683 & 1.2972 & 0.7367 & 0.084 \\
H(8B) & $2 i$ & 0.6188 & 1.2487 & 0.8027 & 0.084 \\
H(8C) & $2 i$ & 0.4735 & 1.1943 & 0.7662 & 0.084 \\
H(13A) & $2 i$ & 0.5966 & 0.8838 & 0.9892 & 0.087 \\
H(13B) & $2 i$ & 0.7006 & 0.9722 & 0.9545 & 0.087 \\
H(13C) & $2 i$ & 0.6874 & 0.8354 & 0.9372 & 0.087 \\
H(14A) & $2 i$ & 0.4411 & 0.6250 & 0.8840 & 0.095 \\
H(14B) & $2 i$ & 0.5876 & 0.6681 & 0.8670 & 0.095 \\
H(14C) & $2 i$ & 0.4748 & 0.6053 & 0.8052 & 0.095 \\
H(15A) & $2 i$ & 0.2070 & 0.6482 & 0.7602 & 0.093 \\
H(15B) & $2 i$ & 0.3314 & 0.6197 & 0.7282 & 0.093 \\
H(15C) & $2 i$ & 0.2584 & 0.7031 & 0.6942 & 0.093 \\
H(16A) & $2 i$ & 0.1798 & 0.9337 & 0.7776 & 0.079 \\
& & & & & \\
& & & & &
\end{tabular}

Table 2. Continued.

\begin{tabular}{|c|c|c|c|c|c|}
\hline Atom & Site & $x$ & $y$ & $z$ & $U_{\text {iso }}$ \\
\hline$H(16 B)$ & $2 i$ & 0.2318 & $0.889 !$ & 0.7065 & 0.079 \\
\hline$H(16 C)$ & $2 i$ & 0.2869 & 1.0215 & 0.7442 & 0.079 \\
\hline$H(17 A)$ & $2 i$ & 0.6571 & 0.2679 & 0.5875 & 0.062 \\
\hline$H(17 B)$ & $2 i$ & 0.6512 & 0.3622 & 0.5376 & 0.062 \\
\hline$H(18 A)$ & $2 i$ & 0.7084 & 0.2236 & 0.4457 & 0.064 \\
\hline$H(18 B)$ & $2 i$ & 0.7261 & 0.1389 & 0.5001 & 0.064 \\
\hline$H(19 A)$ & $2 i$ & 0.9536 & 0.1790 & 0.4826 & 0.065 \\
\hline$H(19 B)$ & $2 i$ & 0.8600 & 0.1106 & 0.4135 & 0.065 \\
\hline$H(20 A)$ & $2 i$ & 1.0177 & 0.1916 & 0.3373 & 0.062 \\
\hline$H(20 B)$ & $2 i$ & 1.1154 & 0.2517 & 0.4063 & 0.062 \\
\hline$H(21 A)$ & $2 i$ & 1.1630 & 0.3516 & 0.3079 & 0.060 \\
\hline$H(21 B)$ & $2 i$ & 1.0269 & 0.3849 & 0.3114 & 0.060 \\
\hline$H(22 A)$ & $2 i$ & 1.1763 & 0.5916 & 0.3353 & 0.063 \\
\hline$H(22 B)$ & $2 i$ & 1.3031 & 0.5440 & 0.3431 & 0.063 \\
\hline$H(23 A)$ & $2 i$ & 1.0371 & 0.3101 & 0.6207 & 0.081 \\
\hline $\mathrm{H}(23 \mathrm{~B})$ & $2 i$ & 1.1390 & 0.4150 & 0.6723 & 0.081 \\
\hline$H(24 A)$ & $2 i$ & 1.1905 & 0.2078 & 0.5998 & 0.116 \\
\hline$H(24 B)$ & $2 i$ & 1.2360 & 0.2653 & 0.6804 & 0.116 \\
\hline$H(25 A)$ & $2 i$ & 1.4000 & 0.3060 & 0.5874 & 0.096 \\
\hline$H(25 B)$ & $2 i$ & 1.4055 & 0.4066 & 0.6528 & 0.096 \\
\hline$H(26 A)$ & $2 i$ & 1.3464 & 0.5139 & 0.5724 & 0.096 \\
\hline$H(26 B)$ & $2 i$ & 1.3074 & 0.4020 & 0.5114 & 0.096 \\
\hline$H(27 A)$ & $2 i$ & 1.1868 & 0.8090 & 0.8920 & 0.062 \\
\hline$H(27 B)$ & $2 i$ & 1.0860 & 0.8132 & 0.9489 & 0.062 \\
\hline$H(28 A)$ & $2 i$ & 1.2534 & 0.8014 & 1.0422 & 0.058 \\
\hline $\mathrm{H}(28 \mathrm{~B})$ & $2 i$ & 1.3516 & 0.8048 & 0.9835 & 0.058 \\
\hline$H(29 A)$ & $2 i$ & 1.3676 & 0.6152 & 0.9992 & 0.061 \\
\hline H(29B) & $2 i$ & 1.4215 & 0.7096 & 1.0685 & 0.061 \\
\hline$H(30 A)$ & $2 i$ & 1.3822 & 0.5512 & 1.1336 & 0.057 \\
\hline $\mathrm{H}(30 \mathrm{~B})$ & $2 i$ & 1.3166 & 0.4567 & 1.0657 & 0.057 \\
\hline$H(31 A)$ & $2 i$ & 1.2371 & 0.4001 & 1.1740 & 0.064 \\
\hline$H(31 B)$ & $2 i$ & 1.1720 & 0.5075 & 1.1806 & 0.064 \\
\hline$H(32 A)$ & $2 i$ & 0.9570 & 0.3701 & 1.1705 & 0.066 \\
\hline H(32B) & $2 i$ & 1.0277 & 0.2664 & 1.1659 & 0.066 \\
\hline $\mathrm{H}(33 \mathrm{~A})$ & $2 i$ & 0.8769 & 0.6151 & 1.1816 & 0.077 \\
\hline H(33B) & $2 i$ & 0.7855 & 0.5144 & 1.1232 & 0.077 \\
\hline$H(34 A)$ & $2 i$ & 0.6444 & 0.6246 & 1.1032 & 0.114 \\
\hline$H(34 B)$ & $2 i$ & 0.6893 & 0.6724 & 1.1851 & 0.114 \\
\hline$H(35 A)$ & $2 i$ & 0.8302 & 0.8352 & 1.1647 & 0.115 \\
\hline H(35B) & $2 i$ & 0.7279 & 0.8149 & 1.0966 & 0.115 \\
\hline$H(36 A)$ & $2 i$ & 0.8656 & 0.7560 & 1.0249 & 0.085 \\
\hline$H(36 B)$ & $2 i$ & 0.9772 & 0.8142 & 1.0887 & 0.085 \\
\hline
\end{tabular}

Table 3. Atomic coordinates and displacement parameters (in $\AA^{2}$ ).

\begin{tabular}{|c|c|c|c|c|c|c|c|c|c|c|}
\hline Atom & Site & $x$ & $y$ & $z$ & $U_{11}$ & $U_{22}$ & $U_{33}$ & $U_{12}$ & $U_{13}$ & $U_{23}$ \\
\hline Nd & $2 i$ & $0.61753(5)$ & $0.91132(5)$ & $0.74440(3)$ & $0.0382(3)$ & $0.0441(3)$ & $0.0417(3)$ & $0.0040(2)$ & $0.0128(2)$ & $0.0137(2)$ \\
\hline $\mathbf{K}(1)$ & $1 \mathrm{~g}$ & 0 & $1 / 2$ & $1 / 2$ & $0.064(2)$ & $0.068(2)$ & $0.063(2)$ & $0.009(2)$ & $0.016(2)$ & $0.015(2)$ \\
\hline$K(2)$ & lc & 0 & $1 / 2$ & 0 & $0.059(2)$ & $0.067(2)$ & $0.058(2)$ & $0.011(2)$ & $0.016(2)$ & $0.017(2)$ \\
\hline $\mathbf{P}(1)$ & $2 i$ & $0.5046(3)$ & $1.0345(3)$ & $0.6393(1)$ & $0.044(2)$ & $0.055(2)$ & $0.044(2)$ & $0.004(1)$ & $0.008(1)$ & $0.017(1)$ \\
\hline $\mathbf{P}(2)$ & $2 i$ & $0.4770(3)$ & $1.0016(2)$ & $0.8628(1)$ & $0.049(2)$ & $0.051(2)$ & $0.043(2)$ & $0.006(1)$ & $0.013(1)$ & $0.013(1)$ \\
\hline$O(1)$ & $2 i$ & $0.8155(6)$ & $0.3089(6)$ & $0.5368(4)$ & $0.043(4)$ & $0.052(4)$ & $0.054(4)$ & $0.000(3)$ & $0.016(3)$ & $0.005(4)$ \\
\hline$O(2)$ & $2 i$ & $0.9370(6)$ & $0.2794(6)$ & $0.4106(4)$ & $0.039(4)$ & $0.060(4)$ & $0.048(4)$ & $0.004(3)$ & $0.012(3)$ & $0.012(4)$ \\
\hline$O(3)$ & $2 i$ & $1.1584(6)$ & $0.4651(6)$ & $0.3942(3)$ & $0.045(4)$ & $0.057(4)$ & $0.042(4)$ & $0.005(3)$ & $0.017(3)$ & $0.014(3)$ \\
\hline$O(4)$ & $2 i$ & $1.1616(8)$ & $0.4172(8)$ & $0.5700(5)$ & $0.058(5)$ & $0.103(7)$ & $0.079(6)$ & $0.001(5)$ & $0.021(4)$ & $0.035(5)$ \\
\hline$O(6)$ & $2 i$ & $1.1765(6)$ & $0.6865(6)$ & $0.9525(3)$ & $0.040(4)$ & $0.049(4)$ & $0.052(4)$ & $0.003(3)$ & $0.009(3)$ & $0.015(4)$ \\
\hline$O(7)$ & $2 i$ & $1.2487(6)$ & $0.5993(6)$ & $1.0750(3)$ & $0.036(4)$ & $0.049(4)$ & $0.052(4)$ & $0.002(3)$ & $0.013(3)$ & $0.008(3)$ \\
\hline$O(8)$ & $2 i$ & $1.0895(6)$ & $0.3805(6)$ & $1.1005(3)$ & $0.042(4)$ & $0.051(4)$ & $0.036(4)$ & $-0.004(3)$ & $0.010(3)$ & $0.017(3)$ \\
\hline$O(9)$ & $2 i$ & $0.9053(8)$ & $0.6391(8)$ & $1.0839(4)$ & $0.069(5)$ & $0.076(6)$ & $0.080(6)$ & $0.020(5)$ & $0.030(5)$ & $0.034(5)$ \\
\hline$C(1)$ & $2 i$ & $0.641(1)$ & $0.9881(9)$ & $0.6069(5)$ & $0.044(6)$ & $0.044(6)$ & $0.044(6)$ & $0.000(5)$ & $0.005(5)$ & $0.003(5)$ \\
\hline$C(2)$ & $2 i$ & $0.7593(9)$ & $1.0507(9)$ & $0.6483(5)$ & $0.039(6)$ & $0.058(7)$ & $0.037(6)$ & $-0.004(5)$ & $0.008(5)$ & $0.018(5)$ \\
\hline$C(3)$ & $2 i$ & $0.744(1)$ & $1.1349(9)$ & $0.7069(5)$ & $0.066(7)$ & $0.052(6)$ & $0.037(6)$ & $0.012(6)$ & $0.017(5)$ & $0.015(5)$ \\
\hline C(4) & $2 i$ & $0.610(1)$ & $1.1365(9)$ & $0.7086(5)$ & $0.046(6)$ & $0.054(6)$ & $0.038(5)$ & $0.012(5)$ & $0.010(5)$ & $0.010(5)$ \\
\hline$C(5)$ & $2 i$ & $0.630(1)$ & $0.8987(9)$ & $0.5406(5)$ & $0.055(6)$ & $0.055(6)$ & $0.036(6)$ & $-0.001(5)$ & $0.010(5)$ & $0.014(5)$ \\
\hline$C(6)$ & $2 i$ & $0.895(1)$ & $1.041(1)$ & $0.6282(6)$ & $0.042(6)$ & $0.057(7)$ & $0.061(7)$ & $0.005(5)$ & $0.020(5)$ & $0.018(6)$ \\
\hline$C(7)$ & $2 i$ & $0.858(1)$ & $1.2178(9)$ & $0.7560(5)$ & $0.066(7)$ & $0.043(6)$ & $0.049(6)$ & $0.003(6)$ & $0.004(6)$ & $0.012(5)$ \\
\hline$C(8)$ & $2 i$ & $0.563(1)$ & $1.2276(9)$ & $0.7581(6)$ & $0.062(7)$ & $0.046(6)$ & $0.062(7)$ & $0.014(6)$ & $0.007(6)$ & $0.012(6)$ \\
\hline$C(9)$ & $2 i$ & $0.536(1)$ & $0.8837(9)$ & $0.8841(5)$ & $0.047(6)$ & $0.045(6)$ & $0.043(6)$ & $0.005(5)$ & $0.016(5)$ & $0.012(5)$ \\
\hline
\end{tabular}


Table 3. Continued

\begin{tabular}{|c|c|c|c|c|c|c|c|c|c|c|}
\hline Alom & Site & $x$ & $y$ & $z$ & $U_{11}$ & $U_{22}$ & $U_{33}$ & $U_{12}$ & $U_{13}$ & $U_{23}$ \\
\hline$C(10)$ & $2 i$ & $0.474(1)$ & $0.7768(9)$ & $0.8409(6)$ & $0.044(6)$ & $0.054(7)$ & $0.049(6)$ & $0.002(5)$ & $0.014(5)$ & $0.016(6)$ \\
\hline $\mathrm{C}(11)$ & $2 i$ & $0.375(1)$ & $0.7884(9)$ & $0.7884(5)$ & $0.040(6)$ & $0.053(7)$ & $0.043(6)$ & $-0.004(5)$ & $0.019(5)$ & $0.012(5)$ \\
\hline $\mathrm{C}(12)$ & $2 i$ & $0.365(1)$ & $0.9064(9)$ & $0.7943(5)$ & $0.053(6)$ & $0.047(6)$ & $0.043(6)$ & $0.007(5)$ & $0.024(5)$ & $0.012(5)$ \\
\hline$C(13)$ & $2 i$ & $0.640(1)$ & $0.895(1)$ & $0.9472(5)$ & $0.060(7)$ & $0.068(8)$ & $0.047(6)$ & $0.011(6)$ & $0.006(6)$ & $0.021(6)$ \\
\hline$C(14)$ & $2 i$ & $0.496(1)$ & $0.658(1)$ & $0.8501(6)$ & $0.061(7)$ & $0.067(8)$ & $0.065(7)$ & $0.014(6)$ & $0.015(6)$ & $0.021(6)$ \\
\hline$C(15)$ & $2 i$ & $0.284(1)$ & $0.680(1)$ & $0.7380(6)$ & $0.046(6)$ & $0.072(8)$ & $0.064(7)$ & $0.007(6)$ & $0.016(6)$ & $0.010(6)$ \\
\hline$C(16)$ & $2 i$ & $0.256(1)$ & $0.9409(9)$ & $0.7517(6)$ & $0.047(6)$ & $0.052(6)$ & $0.057(7)$ & $0.003(5)$ & $0.003(5)$ & $0.018(5)$ \\
\hline$C(17)$ & $2 i$ & $0.709(1)$ & $0.3369(9)$ & $0.5707(6)$ & $0.038(6)$ & $0.056(7)$ & $0.064(7)$ & $0.004(5)$ & $0.027(5)$ & $0.025(6)$ \\
\hline$C(18)$ & $2 i$ & $0.771(1)$ & $0.2079(9)$ & $0.4807(6)$ & $0.048(6)$ & $0.049(6)$ & $0.056(7)$ & $-0.006(5)$ & $0.008(5)$ & $0.016(6)$ \\
\hline$C(19)$ & $2 i$ & $0.886(1)$ & $0.1849(9)$ & $0.4467(6)$ & $0.057(7)$ & $0.051(6)$ & $0.047(6)$ & $-0.001(6)$ & $0.009(5)$ & $0.012(5)$ \\
\hline$C(20)$ & $2 i$ & $1.046(1)$ & $0.262(1)$ & $0.3733(5)$ & $0.046(6)$ & $0.058(7)$ & $0.044(6)$ & $0.003(5)$ & $0.011(5)$ & $0.004(5)$ \\
\hline$C(21)$ & $2 i$ & $1.098(1)$ & $0.3673(9)$ & $0.3393(5)$ & $0.053(6)$ & $0.061(7)$ & $0.033(5)$ & $0.012(6)$ & $0.008(5)$ & $0.003(5)$ \\
\hline $\mathrm{C}(22)$ & $2 i$ & $1.233(1)$ & $0.5654(9)$ & $0.3683(5)$ & $0.064(7)$ & $0.065(7)$ & $0.043(6)$ & $0.022(6)$ & $0.033(5)$ & $0.031(6)$ \\
\hline$C(23)$ & $2 i$ & $1.128(1)$ & $0.358(1)$ & $0.6288(6)$ & $0.058(7)$ & $0.090(9)$ & $0.048(7)$ & $-0.002(7)$ & $0.020(6)$ & $0.015(7)$ \\
\hline$C(26)$ & $2 i$ & $1.298(1)$ & $0.431(1)$ & $0.5604(7)$ & $0.061(8)$ & $0.10(1)$ & $0.067(8)$ & $-0.007(8)$ & $0.011(7)$ & $0.028(8)$ \\
\hline$C(27)$ & $2 i$ & $1.120(1)$ & $0.7607(9)$ & $0.9157(6)$ & $0.047(6)$ & $0.042(6)$ & $0.070(7)$ & $0.011(5)$ & $0.020(6)$ & $0.021(6)$ \\
\hline$C(28)$ & $2 i$ & $1.285(1)$ & $0.7530(9)$ & $1.0055(6)$ & $0.042(6)$ & $0.046(6)$ & $0.057(6)$ & $0.006(5)$ & $0.012(5)$ & $0.015(5)$ \\
\hline$C(29)$ & $2 i$ & $1.343(1)$ & $0.6676(9)$ & $1.0366(6)$ & $0.044(6)$ & $0.053(6)$ & $0.048(6)$ & $-0.002(5)$ & $0.004(5)$ & $0.003(5)$ \\
\hline$C(30)$ & $2 i$ & $1.300(1)$ & $0.5132(9)$ & $1.1038(5)$ & $0.041(6)$ & $0.051(6)$ & $0.049(6)$ & $0.006(5)$ & $0.005(5)$ & $0.018(5)$ \\
\hline$C(31)$ & $2 i$ & $1.199(1)$ & $0.450(1)$ & $1.1472(6)$ & $0.056(7)$ & $0.053(7)$ & $0.052(6)$ & $0.012(6)$ & $0.012(6)$ & $0.012(5)$ \\
\hline$C(32)$ & $2 i$ & $0.990(1)$ & $0.316(1)$ & $1.1383(6)$ & $0.057(7)$ & $0.058(7)$ & $0.048(6)$ & $0.002(6)$ & $0.010(5)$ & $0.025(6)$ \\
\hline$C(33)$ & $2 i$ & $0.825(1)$ & $0.599(1)$ & $1.1356(6)$ & $0.065(8)$ & $0.066(8)$ & $0.049(7)$ & $-0.005(7)$ & $0.001(6)$ & $0.012(6)$ \\
\hline$C(34)$ & $2 i$ & $0.719(1)$ & $0.664(1)$ & $1.1383(9)$ & $0.067(9)$ & $0.09(1)$ & $0.12(1)$ & $0.011(8)$ & $0.023(9)$ & $0.02(1)$ \\
\hline$C(35)$ & $2 i$ & $0.789(2)$ & $0.781(1)$ & $1.1215(9)$ & $0.12(1)$ & $0.09(1)$ & $0.11(1)$ & $0.07(1)$ & $0.03(1)$ & $0.023(9)$ \\
\hline$C(36)$ & $2 i$ & $0.893(1)$ & $0.755(1)$ & $1.0743(7)$ & $0.065(8)$ & $0.076(9)$ & $0.068(8)$ & $0.010(7)$ & $0.002(7)$ & $0.018(7)$ \\
\hline $\mathbf{B}(1)$ & $2 i$ & $0.590(1)$ & $0.7044(9)$ & $0.6611(5)$ & $0.033(6)$ & $0.030(5)$ & $0.038(6)$ & $0.005(5)$ & $0.016(5)$ & $-0.002(5)$ \\
\hline $\mathrm{B}(2)$ & $2 i$ & $0.851(1)$ & $0.932(1)$ & $0.8123(6)$ & $0.035(6)$ & $0.044(7)$ & $0.044(6)$ & $0.007(5)$ & $-0.001(5)$ & $0.012(5)$ \\
\hline
\end{tabular}

\section{References}

1. Barbier-Baudry, D; Blacque, O.; Hafid, A.; Nyassi, A.; Sitzmann, H.; Visseaux, M.: Synthesis and $\mathrm{X}$-ray crystal structures of $\left(\mathrm{C}_{5} \mathrm{H}^{i} \mathrm{Pr}\right)$ $\mathrm{Ln}\left(\mathrm{BH}_{4}\right)_{2}$ (THF) $(\mathrm{Ln}=\mathrm{Nd}$ and $\mathrm{Sm})$, versatile precursors for polymerization catalysts. Eur. J. Inorg. Chem. (2000) 2333-2336.

2. Cendrowski-Guillaume, S. M.; Le Gland, G.; Nierlich, M.; Ephritikhine, M.: Lanthanide borohydrides as precursors to organometallic compounds. Mono(cyclooctatetraenyl) neodymium complexes. Organometallics 19 (2000) 5654-5660.

3. Qian, C.; Nie, W.; Sun, J.: The first example of a dinuclear anionic lanthanoi-

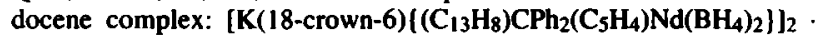
$\mathrm{C}_{4} \mathrm{H}_{8} \mathrm{O}_{2}$. J. Organomet. Chem. 626 (2001) 171-175.

4. Deng, D; Zheng, X.; Qian, C; Sun, J.; Zhang, L.: Studies on organolanthanide complexes XLVII. Syntheses of bis(2-methoxyethylcyclopentadienyl) lanthanide tetrahydroborates ( $\mathrm{Ln}=\mathrm{La}, \mathrm{Pr}, \mathrm{Nd}, \mathrm{Sm}$ or Gd); crystal structures of bis(2-methoxyethylcyclopentadienyl)praseodimium and neodymium tetrahydroborates. J. Organomet. Chem. 466 (1994) 95-100.

5. Cendrowski-Guillaume, S. M.; Nierlich, M.; Lance, M.; Ephritikhine, M.: First chemical transformations of lanthanide borohydride compounds: Synthesis and crystal structures of $\left[\left(\eta-\mathrm{C}_{8} \mathrm{H}_{8}\right) \mathrm{Nd}\left(\mathrm{BH}_{4}\right)(\mathrm{THF})_{2}\right.$ and $\left[\left(\eta-\mathrm{C}_{8} \mathrm{H}_{8}\right) \mathrm{Nd}(\mathrm{THF})_{4}\right]\left[\mathrm{BPh}_{4}\right]$. Organometallics 17 (1998) 786-788.
6. Cendrowski-Guillaume, S. M.; Nierlich, M.; Ephritikhine, M.: Synthesis and $\mathrm{X}$-ray crystal structure of $\left[\mathrm{U}\left(\eta-\mathrm{C}_{8} \mathrm{H}_{8}\right)(\mathrm{HMPA})_{3}\right]\left[\mathrm{BPh}_{4}\right]$, the first cationic monocyclooctatetraenyl compound of uranium(III) and its neodymium homologue. Eur. J. Inorg. Chem. (2001) 1495-1498.

7. Nief, F.: Complexes containing bonds between group 3, lanthanide or actinide metals and non-first-row main group elements (excluding halogens). Coord. Chem. Rev. 178-180 (1998) 13-81.

8. Nief, F.; Mathey, F.: A new application of the tetramethylphospholyl $\left(\eta^{5}-\mathrm{C}_{4} \mathrm{Me} 4 \mathrm{P}\right) \pi$-ligand. Synthesis of $\eta 5$-tetramethylphospholyl complexes of yttrium and lutetium. J. Chem. Commun., Chem. Soc. (1989) 800-801.

9. Nief, F.; Riant, P.; Ricard, L.; Desmurs, P.; Baudry-Barbier, D.: Synthesis and reactivity of bis(phospholyl)neodymium(III) and -samarium(III) chlorides and alkyl derivatives. Eur. J. Inorg. Chem. (1999) 1041-1045.

10. Marks, T. J.; Kolb, J. R.: Covalent transition metal, lanthanide and actinide tetrahydroborate complexes. Chem. Rev. 77 (1977) 263-293.

11. Makhaev, V. D.: Structural and dynamic properties of tetrahydroborate complexes. Russian Chem. Rev. 69 (2000) 727-746.

12. Sheldrick, G. M.: SHELXTL, version 5.1, Bruker-AXS, Madison, Wisconsin, USA 1999. 